\title{
SOME COUNTEREXAMPLES CONCERNING A DIFFERENTIAL CRITERION FOR FLATNESS
}

\author{
WILLIAM C. BROWN AND SARAH GLAZ
}

\begin{abstract}
Let $A$ denote a commutative ring with identity. We suppose $A$ contains a field $k$ of characteristic zero. Let $\Omega_{k}^{1}(A)$ and $d: A \rightarrow \Omega_{k}^{1}(A)$ denote the $A$-module of first-order $k$-differentials on $A$ and the canonical derivation of $A$ into $\Omega_{k}^{1}(A)$ respectively. If $\mathscr{A}$ is an ideal of $A$ which is flat as an $A$-module, then $x d y-y d x \in$ $\mathfrak{Y}^{2} \Omega_{k}^{1}(A)$ for all $x, y$ in $\mathfrak{A}$. We give examples in this paper which show that the converse of this statement is false. We also show that if $\mathfrak{A}$ is a maximal ideal of a Noetherian ring $A$, then $x d y-y d x \in \mathfrak{A}^{2} \Omega_{k}^{1}(A)$ for all $x, y$ in $\mathfrak{A}$ does imply $\mathfrak{A}$ is flat.
\end{abstract}

1. Introduction. Throughout this paper, $A$ will denote a commutative ring with identity. We shall assume that $A$ contains a field $k$ of characteristic zero. We shall let $\Omega_{k}^{1}(A)$ denote the $A$-module of first-order $k$-differentials of $A$. Thus, $\Omega_{k}^{1}(A)=$ $I / I^{2}$, where $I$ denotes the kernel of the multiplication map $\mu: A \otimes_{k} A \rightarrow A$ which is given by $\mu\left(\sum a_{i} \otimes_{k} a_{i}^{\prime}\right)=\sum a_{i} a_{i}^{\prime}$. We shall let $d: A \rightarrow \Omega_{k}^{1}(A)$ denote the canonical $k$-derivation on $A$. If $x$ and $y$ are two elements of $A$, we set $\Delta_{d}(x, y)=x d(y)-$ $y d(x)$. If $\mathfrak{A}$ is an ideal of $A$, and $x, y \in \mathfrak{A}$, then clearly $\Delta_{d}(x, y) \in \mathfrak{A} \Omega_{k}^{1}(A)$. We shall say that $\mathfrak{A}$ satisfies condition $(D)$ if $\Delta_{d}(x, y) \in \mathfrak{A}^{2} \Omega_{k}^{1}(A)$ for all $x, y \in \mathfrak{A}$. It is a well-known fact that if $\mathfrak{U}$ is a flat ideal of $A$; i.e. if $\mathfrak{A}$ is a flat $A$-module, then $\mathfrak{A}$ satisfies condition $(D)$. A proof of this remark can be found in [4].

In [1], the converse question was explored. That is, when does condition $(D)$ imply $\mathfrak{A}$ is a flat ideal? In [1], it was shown that if $A$ is a regular local ring containing a field $k$, then every ideal $\mathfrak{A}$ in $A$ which satisfies condition $(D)$ is flat. If $A$ is not regular, then the question of when condition $(D)$ implies flatness is much harder to study. The best result obtained in [1] is as follows: Suppose $(A, m, k)$ denotes a complete, local domain of dimension one with maximal ideal $m$ and residue class field $k$. Suppose $A$ is a monomial ring, i.e. a ring of the form $A=k\left[\left[t^{a_{1}}, \ldots, t^{a_{1}}\right]\right]$ with $k[[t]]$ being the integral closure of $A$. Then any ideal $\mathfrak{A}$ in $A$ satisfying condition $(D)$ is flat.

In view of the above-mentioned result and other evidence collected in [1], it seemed reasonable that the following conjecture (made in [1]) was true: Let $(A, m, k)$ denote a one-dimensional local domain, essentially of finite type over $k$. If $\mathfrak{A}$ is an ideal of $A$ which satisfies condition $(D)$, then $\mathfrak{A}$ is flat. In this paper, we shall give an example which shows that this conjecture is false. We shall also give an example of a complete local domain of dimension one which is not monomial and contains nonflat ideals satisfying condition $(D)$. We shall show that if $A$ is a

Received by editors May 1, 1980.

AMS (MOS) subject classifications (1970). Primary 13C10; Secondary $13 \mathrm{~B} 10$.

Key words and phrases. Condition (D), monomial ring, differentials, flatness.

(c) 1981 American Mathematical Society 0002-9939/81/0000-0150/\$02.50 
Noetherian ring which contains $k$ and $p$ a prime ideal of $A$ satisfying condition $(D)$, then $p$ must be a regular prime of height less than or equal to one, but $p$ need not be flat.

2. Some examples We first give an example which shows that the conjecture mentioned in the introduction is false.

EXAMPLE I. Let $k$ be an arbitrary field of characteristic zero, Let $A$ denote the local ring at the origin of the plane curve $C: Y^{2}=X^{2}(X+1)$ in $\mathbf{A}_{k}^{2}$. Thus, $A$ can be written in the form $A=k[x, y]_{(x, y)}$. Here $y^{2}=x^{2}(x+1)$, and $k[x, y]_{(x, y)}$ denotes the finitely-generated, integral domain $k[x, y]$ localized at the maximal ideal $(x, y)$. We set $\mathfrak{A}=\left(x^{2}, x y\right)$. We note that $\mathfrak{A}$ is a stable ideal with transversal $x^{2}$ (see [3] for definitions). If $\mathfrak{A}$ is flat and, consequently, principal since $A$ is local, then $\mathfrak{A}=\left(x^{2}\right)$. But then $y=\lambda x$ for some $\lambda \in A$. This implies $A$ is a regular local ring which is not the case. Thus, $\mathfrak{A}$ is not flat. We shall show that $\mathfrak{A}$ satisfies condition $(D)$.

If $A$ is any ring containing $k$, and $\mathfrak{A}=(a, b)$ is any two-generated ideal of $A$, then one easily sees that $\mathfrak{A}$ satisfies condition $(D)$ if and only if $\Delta_{d}(a, b) \in$ $\mathfrak{A}^{2} \Omega_{k}^{1}(A)$. Thus, in our example, we must show $\Delta_{d}\left(x^{2}, x y\right) \in\left(x^{4}, x^{3} y\right) \Omega_{k}^{1}(A)$. Now $\Delta_{d}\left(x^{2}, x y\right)=x^{3} d y-x^{2} y d x$. Since $x+1$ is a unit in $A, x^{2}=(x+1)^{-1} y^{2}$. Also, $y^{2}=x^{2}+x^{3}$ implies $2 y d y=\left(3 x^{2}+2 x\right) d x$. Making these substitutions in $x^{3} d y-$ $x^{2} y d x$, we get $\Delta_{d}\left(x^{2}, x y\right)=[2(x+1)]^{-1} x^{3} y d x \in \mathfrak{A}^{2} \Omega_{k}^{1}(A)$.

If we pass to the completion $\hat{A}$ of $A$ in Example I and consider the ideal $\hat{\mathfrak{A}}=\mathfrak{A} \hat{A}$, then $\hat{\mathfrak{A}}$ satisfies condition $(D)$ but is not flat. $\hat{A}$ is a complete local ring of dimension one. If $k$ is algebraically closed, then $\hat{A}$ is not an integral domain ( $C$ is not unibranched). On the other hand, if $k$ is the rational numbers, then $\hat{A}$ is a domain. In attempting to get rid of this example by putting more hypotheses on $\hat{A}$, we are led naturally to the following problem: Let the triple $(A, m, k)$ denote a complete local domain $A$ of Krull dimenson one, with maximal ideal $m$ and residue class field $k$. We assume $k$ is an algebraically closed field of characteristic zero which is contained in $A$. Suppose $\mathfrak{A}$ is an ideal of $A$ which satisfies condition $(D)$. Is $\mathfrak{A}$ flat? The answer to this question is no as our next example will show.

EXAMPLE II. Let $k$ be any field of characteristic zero. Let $t$ be an indeterminate over $k$ and consider the formal power-series ring $\bar{A}=k[[t]] . \bar{A}$ is a discrete, rank-one valuation ring with valuation $\nu: \bar{A} \rightarrow \mathbf{Z}^{+} \cup\{0, \infty\}$ given by $\nu(t)=1$. Set $h_{1}=t^{5}-(5 / 16) t^{8}, h_{2}=(16 / 5) t^{11}+(8 / 5) t^{14}$ and $h_{3}=t^{16}-(2 / 11) t^{22}+t^{23}$. We now consider the subring $A$ of $\bar{A}$ which consists of all power-series (coefficients in $k$ ) in the elements $h_{1}, h_{2}, h_{3}$ and $t^{i}$ for $i \geqslant 24$. Thus,

$$
A=k\left[\left[h_{1}, h_{2}, h_{3}, t^{24}, t^{25}, \ldots\right]\right]
$$

Since any semigroup of positive integers is finitely generated, we see that $A$ has the form $A=k\left[\left[h_{1}, h_{2}, h_{3}, t^{24}, t^{25}, \ldots, t^{r}\right]\right]$ for some $r$ sufficiently large. Thus, $A$ is a complete local domain of dimension one. The quotient field of $A$ is clearly $k((t))$ : the quotient field of $\bar{A} ; \bar{A}$ is the integral closure of $A$ in $k((t))$ and $k$ is the residue-class field of $A$. Set $m=\left(h_{1}, h_{2}, h_{3}, t^{24}, \ldots, t^{r}\right) ; m$ is clearly the maximal 
ideal of $A$ and $(A, m, k)$ forms a triple as discussed above, provided we take $k$ algebraically closed.

Let $\nu(A)=\{\nu(x) \mid x \in A\}$ denote the value semigroup of $A$. We claim that $23 \notin \nu(A)$. To see this, we proceed as follows: If $23 \in \nu(A)$, then $23=\nu(g)$ for some $g \in A$. We could then write $g=\alpha_{1} h_{1}+\alpha_{2} h_{2}+\alpha_{3} h_{3}+\alpha_{4} h_{1}^{2}+\alpha_{5} h_{2}^{2}+$ $\alpha_{6} h_{1} h_{2}+\alpha_{7} h_{1} h_{3}+\alpha_{8} h_{1}^{3}+\alpha_{9} h_{1}^{2} h_{2}+\alpha_{10} h_{1}^{4}+\gamma t^{24}$ with $\alpha_{1}, \alpha_{2}, \ldots, \alpha_{10} \in k$, and $\gamma$ $\in \bar{A}$. A simple arithmetic computation, carefully noting the $\nu$-values of these summands, shows that this is impossible. Therefore, $23 \notin \nu(A)$.

Now set $\mathfrak{A}=\left(t^{24}, t^{47}\right) \subset A$. We note that no nonzero multiple $x \mathfrak{U}$ of $\mathfrak{A}$ is flat. For suppose there exists a nonzero element $x$ of $A$ such that $x \mathfrak{A}$ is flat. Then $x \mathfrak{A}=\left(x t^{24}, x t^{47}\right)$ is principal. Say $\left(x t^{24}, x t^{47}\right)=(y)$. The smallest value any element in $x \mathfrak{A}$ can have is $\nu(x)+24$. Therefore, $\nu(y)=\nu(x)+24$. But then $x t^{47}=\lambda y$ for some $\lambda$ in $A$ implies $23 \in \nu(A)$. Thus $x \mathfrak{U}$ is not flat for any $x \neq 0$. We shall complete Example II by arguing that some nonzero multiple $x \mathfrak{U}$ of $\mathfrak{A}$ satisfies condition $(D)$.

Let $\partial / \partial t$ denote the usual first-order $k$-derivation on $\bar{A}$ given by $(\partial / \partial t)(t)=1$. Set $M=A \partial A / \partial t$, the $A$-submodule $\sim($ of $\bar{A})$ generated by the image of $A$ under $\partial / \partial t$. An easy computation shows that $\left(\partial h_{1} / \partial t\right) h_{2}-\partial h_{3} / \partial t=-23 t^{22}$. This implies $\Delta_{\partial / \partial t}\left(t^{24}, t^{47}\right)=t^{24}(\partial / \partial t)\left(t^{47}\right)-t^{47}(\partial / \partial t)\left(t^{24}\right) \in \mathfrak{A}^{2} M$.

Now let $\hat{\Omega}_{k}^{1}(A)$ and $\hat{\Omega}_{k}^{1}(\bar{A})$ denote the separated completions of $\Omega_{k}^{1}(A)$ and $\Omega_{k}^{1}(\bar{A})$, respectively. Thus, $\hat{\Omega}_{k}^{1}(A)=\Omega_{k}^{1}(A) / \cap_{i=1}^{\infty} m^{i} \Omega_{k}^{1}(A)$ and $\hat{\Omega}_{k}^{1}(\bar{A})=$ $\Omega_{k}^{1}(\bar{A}) / \cap_{i=1}^{\infty} t^{i} \Omega_{k}^{1}(\bar{A})$. It is well known (see e.g. [2, p. 9]) that $\hat{\Omega}_{k}^{1}(\bar{A}) \cong \bar{A}$, and that the kernel of the natural map from $\hat{\Omega}_{k}^{1}(A)$ to $\hat{\Omega}_{k}^{1}(\bar{A})$ is a torsion submodule of $\hat{\Omega}_{k}^{1}(A)$. If we let $\psi: \Omega_{k}^{1}(A) \rightarrow \bar{A}$ be the natural $A$-module homomorphism determined by the composite map $\Omega_{k}^{1}(A) \rightarrow \hat{\Omega}_{k}^{1}(A) \rightarrow \hat{\Omega}_{k}^{1}(\bar{A}) \rightarrow \bar{A}$, then the image of $\psi$ is precisely $M$. The above remarks easily imply that the kernel of $\psi$ is given by ker $\psi=\left\{\gamma \in \Omega_{k}^{1}(A) \mid x \gamma \in \cap_{i=1}^{\infty} m^{i} \Omega_{k}^{1}(A)\right.$ for some $\left.x \in A-\{0\}\right\}$. Since $\Delta_{\partial / \partial t}\left(t^{24}, t^{47}\right) \in \mathfrak{A}^{2} M$, we conclude that there exists a $\gamma_{1} \in \mathfrak{A}^{2} \Omega_{k}^{1}(A)$ and $\gamma_{2} \in \operatorname{ker} \psi$ such that $\Delta_{d}\left(t^{24}, t^{47}\right)=\gamma_{1}+\gamma_{2}$. Since $\gamma_{2} \in \operatorname{ker} \psi$, there exists an $x \in A-\{0\}$ such that $x \gamma_{2} \in \cap_{i=1}^{\infty} m^{i} \Omega_{k}^{1}(A)$. Then $x^{2} \gamma_{2} \in \cap_{i=1}^{\infty} m^{i} \Omega_{k}^{1}(A)$. Since $A$ has Krull dimension one, $m^{l} \subset(x \mathfrak{U})^{2}$ for some $l$ sufficiently large. Thus, $x^{2} \gamma_{2} \in(x \mathfrak{U})^{2} \Omega_{k}^{1}(A)$. Since $\Delta_{d}\left(x t^{24}, x t^{47}\right)=x^{2} \Delta_{d}\left(t^{24}, t^{47}\right)=x^{2} \gamma_{1}+x^{2} \gamma_{2} \in(x \mathfrak{U})^{2} \Omega_{k}^{1}(A)$, we conclude that $x \mathfrak{A}$ satisfies condition $(D)$. Since $x \mathfrak{A}$ is not flat, Example II is now complete.

In light of Example II given here, it is now clear that the positive result for monomial rings mentioned in the introduction to this paper is about as good as one could hope to get.

3. Primes in Noetherian rings. We begin by showing that maximal ideals which satisfy condition $(D)$ must be flat.

TheOREM. Let $A$ denote a Noetherian ring which contains a field $k$ of characteristic zero. Let $m$ be a maximal ideal of $A$. If $m$ satisfies condition (D), then $m$ is flat.

Proof. Let $\hat{A}_{m}$ denote the $m$-adic completion of the local ring $A_{m}$. Since $m$ is a maximal ideal of $A, m$ is flat if and only if $m \hat{A}_{m}$ is a flat $\hat{A}_{m}$-module. The complete local ring $\hat{A}_{m}$ is of equal characteristic and, consequently, contains a copy $L$ of its 
residue-class field. Since the characteristic of $k$ is zero, we can assume $L$ contains $k$. One easily checks that $m \hat{A}_{m}$ satisfies condition $(D)$ in $\hat{A}_{m}$. Since $L \supset k, m \hat{A}_{m}$ satisfies condition $(D)$ with $k$ replaced by $L$. Thus, by replacing $A$ by $\hat{A}_{m}, m$ by $m \hat{A}_{m}$, and $k$ by $L$, we have reduced the theorem to the following claim: Let $A$ be a complete local ring containing a field $k$ of characteristic zero. Suppose $m$ is the maximal ideal of $A$, and $A / m=k$. Then, if $m$ satisfies condition $(D), m$ is flat.

If $A$ is a regular local ring, then the claim follows from [1, Theorem 1.1]. So we can assume $A$ is not regular. In this case, we shall argue that $m$ cannot satisfy condition $(D)$. This contradiction will complete the proof of the claim.

So assume $A$ is not a regular local ring. We first claim that $A$ can be written in the form $A=k\left[\left[Y_{1}, \ldots, Y_{n}\right]\right] / \mathfrak{A}$ with $\mathfrak{A} \subset\left(Y_{1}, \ldots, Y_{n}\right)^{2}$. Here $k\left[\left[Y_{1}, \ldots, Y_{n}\right]\right]$ denotes the formal power-series ring over $k$ in indeterminates $Y_{1}, \ldots, Y_{n}$. To see this, we first note that since $A$ is a complete local ring of equal characteristic, $A$ can be written in the form $A=k\left[\left[X_{1}, \ldots, X_{N}\right]\right] / I$. Here $X_{1}, \ldots, X_{N}$ are indeterminates, and $I$ is some ideal contained in $M=\left(X_{1}, \ldots, X_{N}\right)$. Let $I=$ $\left(f_{1}, \ldots, f_{s}\right)$. If $f_{i} \in M^{2}$ for all $i=1, \ldots, s$, then there is nothing to prove. So, we can assume there exists an integer $g$ such that $1<g<s,\left\{f_{1}, \ldots, f_{g}\right\} \subset M-M^{2}$ and $\left\{f_{g+1}, \ldots, f_{s}\right\} \subset M^{2}$. Since, $f_{1} \in M-M^{2}, f_{1}$ is part of a regular system of parameters for $R=k\left[\left[X_{1}, \ldots, X_{N}\right]\right]$. If $f_{2} \in M^{2}\left(R /\left(f_{1}\right)\right)$, then $f_{2}=F_{1}+z f_{1}$ for some $z \in R$, and $F_{1} \in M^{2}$. If this is the case, we can write $I=$ $\left(f_{1}, f_{3}, \ldots, f_{g}, F_{1}, f_{g+1}, \ldots, f_{s}\right)$ with $f_{1}, f_{3}, \ldots, f_{g} \in M-M^{2}$, and $F_{1}, f_{g+1}, \ldots, f_{s}$ $\in M^{2}$. if $f_{2} \notin M^{2}\left(R /\left(f_{1}\right)\right)$, then $\left\{f_{1}, f_{2}\right\}$ is part of a regular system of parameters of $R$. Continuing this procedure, we see that we can rewrite $I$ as $I=$ $\left(g_{1}, \ldots, g_{N-n}, F_{1}, \ldots, F_{s+n-N}\right)$ with $\left\{g_{1}, \ldots, g_{N-n}\right\}$ part of a regular system of parameters of $R$, and $\left\{F_{1}, \ldots, F_{s+n-N}\right\} \subset M^{2}$. Now suppose $\left\{g_{1}, \ldots, g_{N-n}, g_{N-n+1}, \ldots, g_{N}\right\}$ is a regular system of parameters of $R$. Then $R=k\left[\left[g_{1}, \ldots, g_{N}\right]\right]$. Thus, $A=R / I=k\left[\left[g_{N-n+1}, \ldots, g_{N}\right]\right] / \mathfrak{A}$ where $\mathfrak{A}$ is the image of $\left(F_{1}, \ldots, F_{s+n-N}\right)$ in $k\left[\left[g_{N-n+1}, \ldots, g_{N}\right]\right]$. Setting $Y_{i}=g_{N-n+i}$, for $i=$ $1, \ldots, n$ gives the required result.

For future reference, let $y_{i}=Y_{i}+\mathfrak{A}$. Then $A=k\left[\left[y_{1}, \ldots, y_{n}\right]\right]$, and $m=$ $\left(y_{1}, \ldots, y_{n}\right)$ is the maximal ideal of $A$. Let us relabel the generators of $\mathfrak{A}$ as $\mathfrak{A}=\left(h_{1}, \ldots, h_{r}\right)$. Then $\left\{h_{1}, \ldots, h_{r}\right\} \subset\left(Y_{1}, \ldots, Y_{n}\right)^{2}$. Let $\hat{\Omega}_{k}^{1}(A)$ denote the separated completion of $\Omega_{k}^{1}(A)$. Thus, $\hat{\Omega}_{k}^{1}(A)=\Omega_{k}^{1}(A) / \cap_{i=1}^{\infty} m^{i} \Omega_{k}^{1}(A)$. Let $\chi: \Omega_{k}^{1}(A) \rightarrow$ $\hat{\Omega}_{k}^{1}(A)$ denote the natural projection and set $\hat{d}=\chi \circ d$. Then $\hat{d}$ is a $k$-derivation of $A$ into $\hat{\Omega}_{k}^{1}(A)$. It is well known that $\hat{\Omega}_{k}^{1}(A)=\sum_{i=1}^{n} A \hat{d}\left(y_{i}\right)$. In particular, there exists a surjective $A$-module homorphism $\theta: A^{n} \rightarrow \hat{\Omega}_{k}^{1}(A)$ given by $\theta\left(a_{1}, \ldots, a_{n}\right)=$ $\sum_{i=1}^{n} a_{i} \hat{d}\left(y_{i}\right)$. The kernel of the map $\theta$ is easily seen to be given by ker $\theta=\sum_{i=1}^{r} A \lambda_{i}$ where $\lambda_{i}=\left(\left(\partial h_{i} / \partial Y_{1}\right)^{-}, \ldots,\left(\partial h_{i} / \partial Y_{n}\right)^{-}\right)$for $i=1, \ldots, r$. Here $\left(\partial h_{i} / \partial Y_{j}\right)^{-}$denotes the image of $\partial h_{i} / \partial Y_{j}$ in $A$.

Now let $\Delta_{\hat{d}}\left(a, a^{\prime}\right)=a \hat{d}\left(a^{\prime}\right)-a^{\prime} \hat{d}(a)$ for $a, a^{\prime} \in m$. Since $m$ satisfies condition (D), $\Delta_{\hat{d}}\left(a, a^{\prime}\right) \in m^{2} \hat{\Omega}_{k}^{1}(A)$ for all $a, a^{\prime} \in m$. In particular, $\Delta_{\hat{d}}\left(y_{1}, y_{2}\right) \in$ $\left(y_{1}, \ldots, y_{n}\right)^{2} \hat{\Omega}_{k}^{1}(A)$. Thus, $\Delta_{\dot{d}}\left(y_{1}, y_{2}\right)=\sum_{i j=1}^{n} y_{i} y_{j} \omega_{i j}$ for some $\omega_{i j} \in \hat{\Omega}_{k}^{1}(A)$. Write each $\omega_{i j}$ as $\omega_{i j}=\sum_{t=1}^{n} \bar{a}_{i j t} \hat{d}\left(y_{t}\right)$ with $\bar{a}_{i j t} \in A$. Substituting these expressions into 
$\Delta_{\hat{d}}\left(y_{1}, y_{2}\right)$ gives us the following equation in $\hat{\Omega}_{k}^{1}(A)$ :

$$
\begin{aligned}
\left(-y_{2}-\sum_{i, j=1}^{n} \bar{a}_{i j 1} y_{i} y_{j}\right) \hat{d}\left(y_{1}\right)+ & \left(y_{1}-\sum_{i, j=1}^{n} \bar{a}_{i j 2} y_{i} y_{j}\right) \hat{d}\left(y_{2}\right) \\
& -\sum_{t=3}^{n} \sum_{i, j=1}^{n} \bar{a}_{i j} y_{i} y_{j} \hat{d}\left(y_{t}\right)=0 .
\end{aligned}
$$

Equation (1) implies that the $n$-tuple

$$
\left(-y_{2}-\sum_{i, j=1}^{n} \bar{a}_{i j 1} y_{i} y_{j}, y_{1}-\sum_{i, j=1}^{n} \bar{a}_{i j 2} y_{i} y_{j},-\sum_{i, j=1}^{n} \bar{a}_{i j 3} y_{i} y_{j}, \ldots,-\sum_{i, j=1}^{n} \bar{a}_{i j n} y_{i} y_{j}\right)
$$

lies in the kernel of $\theta$. In particular, there exist elements $\bar{z}_{1}, \ldots, \bar{z}_{r} \in A$ such that the following equations are satisfied:

$$
-y_{2}-\sum_{i, j=1}^{n} \bar{a}_{i j 1} y_{i} y_{j}=\sum_{t=1}^{r} \bar{z}_{t}\left(\frac{\partial h_{t}}{\partial Y_{1}}\right)^{-}, \quad y_{1}-\sum_{i, j=1}^{n} \bar{a}_{i j 2} y_{i} y_{j}=\sum_{t=1}^{r} \bar{z}_{t}\left(\frac{\partial h_{t}}{\partial Y_{2}}\right)^{-} \text {. }
$$

Pulling these equations back to $k\left[\left[Y_{1}, \ldots, Y_{n}\right]\right]$ gives

$$
\begin{array}{r}
-Y_{2}-\sum_{i, j=1}^{n} a_{i j 1} Y_{i} Y_{j}-\sum_{t=1}^{r} z_{t}\left(\frac{\partial h_{t}}{\partial Y_{1}}\right) \in\left(h_{1}, \ldots, h_{r}\right), \\
Y_{1}-\sum_{i j=1}^{n} a_{i j 2} Y_{i} Y_{j}-\sum_{t=1}^{r} z_{t}\left(\frac{\partial h_{t}}{\partial Y_{2}}\right) \in\left(h_{1}, \ldots, h_{r}\right) .
\end{array}
$$

In relation (3), $a_{i j 1}, a_{i j 2}$ and $z_{t}$ are preimages in $k\left[\left[Y_{1}, \ldots, Y_{n}\right]\right]$ of $\bar{a}_{i j 1}, \bar{a}_{i j 2}$ and $\bar{z}_{t}$, respectively. Each $h_{i}$ appearing in relation (3) lies in $\left(Y_{1}, \ldots, Y_{n}\right)^{2}$. So, we can write $h_{i}=\sum_{p, q=1}^{n} \alpha_{p, q, i} Y_{p} Y_{q}+\mu_{i}$ for $i=1, \ldots, r$. Here, $\alpha_{p, q, i} \in k$, and $\mu_{i} \in$ $\left(Y_{1}, \ldots, Y_{n}\right)^{3}$ for all $p, q$ and $i$. If we substitute these expressions for the $h_{i}$ in relation (3) and simplify, we readily see (3) is impossible. Hence, $m$ cannot satisfy condition $(D)$, and the proof of the theorem is now complete.

COROllary. Let $A$ denote a Noetherian ring which contains a field $k$ of characteristic zero. Let $p$ denote a prime ideal of $A$ which satisfies condition $(D)$. Then the local ring $A_{p}$ is regular, and $p$ has height less than or equal to one.

Proof. The same proof as in the theorem implies that $\hat{A}_{p}$ is a regular local ring with $p \hat{A}_{p}$ flat over $\hat{A}_{p}$. Thus, the dimension of $\hat{A}_{p}$ is at most one. Therefore, $A_{p}$ is regular, and height $(p)<1$.

In the corollary, we can conclude that $p \hat{A}_{p}$ is flat. Thus, $p A_{p}$ is also flat over $A_{p}$. However, since $p$ may not be maximal, we cannot conclude that $p$ itself is flat. In fact, $p$ may not be flat as our concluding example shows.

EXAMPLE III. Let $k$ be any field of characteristic zero, and let $X, Y, Z$ and $T$ be indeterminates over $k$. Let $\mathfrak{A}$ be the ideal in $k[X, Y, Z, T]$ which is generated by $X^{2}, Y^{2}, X Y, T Y-Z X$ and $X(T-1)$. Let $A=k[X, Y, Z, T] / \mathfrak{A}$ and as usual let $x, y, z$ and $t$ denote the residues of $X, Y, Z$ and $T$, respectively, in $A$. One easily checks that $p=(x, y)$ is a prime ideal nilpotent of index two in $A$. Clearly $A / p \cong k[Z, T]$. 
We first claim that $p$ satisfies condition (D). To see this, let $d: A \rightarrow \Omega_{k}^{1}(A)$ denote the canonical derivation. Since $p^{2}=0, p$ satisfies $(D)$ if and only if $x d y-y d x=0$ in $\Omega_{k}^{1}(A)$. Now $\Omega_{k}^{1}(A)$ is spanned as an $A$-module by $d x, d y, d z$ and $d t$. We, therefore, have a surjective map $\theta: A^{4} \rightarrow \Omega_{k}^{1}(A)$ given by $\theta\left(a_{1}, a_{2}, a_{3}, a_{4}\right)=a_{1} d x+$ $a_{2} d y+a_{3} d z+a_{4} d t$. From the relations on $A$, we see that the kernel $K$ of $\theta$ contains the four-tuples $(x, 0,0,0),(0, y, 0,0),(y, x, 0,0),(-z, t,-x, y)$ and $(t-$ $1,0,0, x)$. Now $(-y, x, 0,0)=(t-1)(y, x, 0,0)+x(-z, t,-x, y)$ in $A^{4}$. Thus, $(-y, x, 0,0) \in K$. So, $x d y-y d x=0$, and $p$ satisfies condition $(D)$.

We next claim that $p$ is not a flat $A$-module. If $p$ was flat over $A$, then $p \otimes_{A} p \cong p^{2}=0$. In particular, $x \otimes_{A} x=0$ in $p \otimes_{A} p$. We can now apply the usual Bourbaki lemma (e.g. see [5, p. 142]) and conclude that there exist $b_{11}, b_{12}, b_{21}, b_{22} \in A$ such that the following equations are satisfied:
(i) $b_{11} x+b_{21} y=0$,
(iii) $b_{11} x+b_{12} y=x$,
(ii) $b_{12} x+b_{22} y=0$,
(iv) $b_{21} x+b_{22} y=0$.

Now equations (i) and (iii) in (4) imply that $x=c y$ for some $c \in A$. Pulling back to $k[X, Y, Z, T]$, we see this is impossible. Hence, $p$ satisfies condition $(D)$, but is not flat over $A$.

\section{REFERENCES}

1. S. Glaz, Differential criteria for flatness, Proc. Amer. Math. Soc. 79 (1980), 17-22.

2. J. Herzog, Eindimensionale fast-vollständige Durchschnitte sind nicht starr, Manuscripta Math. 30 (1979), 1-19.

3. J. Lipman, Stable ideals and Arf rings, Amer. J. Math. 93 (1971), 649-685.

4. J. D. Sally and W. V. Vasconcelos, Flat ideals. I, Comm. Algebra 3 (1975), 531-543.

5. W. W. Smith Invertible ideals and overrings, Houston J. Math. 5 (1979), 141-153.

Department of Mathematics, Michigan State University, East Lansing, Michigan 48824 (Current address of W. C. Brown)

Current address (Sarah Glaz): Department of Mathematics, Wesleyan University, Middletown, Connecticut 06457 